\title{
Composition of the teat canal and intramammary microbiota of dairy cows subjected to antimicrobial dry cow therapy and internal teat sealant
}

\author{
Hooman Derakhshani, ${ }^{*}$ Jan C. Plaizier, ${ }^{*}$ Jeroen De Buck, $\nmid$ Herman W. Barkema, $\nmid$ and Ehsan Khafipour ${ }^{*} \ddagger^{1}$ \\ ${ }^{*}$ Department of Animal Science, University of Manitoba, Winnipeg, MB, Canada R3T 2N2 \\ †Department of Production Animal Health, Faculty of Veterinary Medicine, University of Calgary, Calgary, AB, Canada \\ ‡Department of Medical Microbiology and Infectious Diseases, University of Manitoba, Winnipeg, MB, Canada
}

\section{ABSTRACT}

Antimicrobial dry cow therapy (DCT) is an important component of mastitis control programs aimed to eliminate existing intramammary infections and prevent the development of new ones during the dry period. However, to what extent the microbiota profiles of different niches of the udder change during the dry period and following administration of DCT remains poorly understood. Therefore, the main objective of the present study was to qualitatively evaluate dynamics of the microbiota of teat canal (TC) and mammary secretions (i.e., milk and colostrum) of healthy udder quarters subjected to DCT using a long-acting antimicrobial product, containing penicillin $\mathrm{G}$ and novobiocin, in combination with internal teat sealant. To this end, TC swabs $(\mathrm{n}=58)$ and their corresponding milk $(\mathrm{n}=$ $29)$ and colostrum samples $(\mathrm{n}=29)$ were collected at the time of drying off and immediately after calving from clinically healthy udder quarters of Holstein dairy cows from a commercial dairy farm. All samples were subjected to DNA extraction and high-throughput sequencing of the V1-V2 hypervariable regions of bacterial 16S rRNA genes. Overall, shifts were more pronounced within the microbiota of mammary secretions than the TC. In particular, microbiota of colostrum samples collected immediately after calving were less species-rich compared with the pre-DCT milk samples. Proportions of several bacterial genera belonging to the phylum Proteobacteria, including Pseudomonas, Stenotrophomonas, and unclassified Alcaligenaceae, were enriched within the microbiota of colostrum samples, whereas Firmicutes genera, including Butyrivibrio, unclassified Clostridiaceae, and unclassified Bacillales, were overrepresented in pre-DCT milk microbiota. Apart from shifts in the proportion of main bacterial genera and phyla, qualitative analysis revealed a high

Received April 2, 2018.

Accepted July 8, 2018.

${ }^{1}$ Corresponding author: Ehsan.Khafipour@umanitoba.ca degree of commonality between pre-DCT and postpartum microbiota of both niches of the udder. Most importantly, a considerable number of bacterial genera and species commonly regarded as mastitis pathogens or opportunists (or both), including Staphylococcus spp., unclassified Enterobacteriaceae, and Corynebacterium spp., were shared between pre-DCT and postpartum microbiota of mammary secretions. Percentage of shared bacterial genera and species was even higher between pre-DCT and postpartum microbiota of TC samples, suggesting that the DCT approach of the present study had limited success in eliminating a considerable proportion of bacteria during the dry period. Key words: teat canal microbiota, intramammary microbiota, dry cow therapy, mastitis

\section{INTRODUCTION}

Intramammary administration of long-acting antimicrobials to every quarter of every cow at drying off, a process known as blanket dry cow therapy (DCT), has proven widely useful in eliminating existing IMI and preventing the development of new IMI throughout the dry period (Bradley and Green, 2004). However, due to growing concerns over development of antimicrobial resistance in mastitis pathogens with zoonotic potential (Oliver et al., 2011; Tang et al., 2017), prophylactic use of antimicrobials in livestock has come under increased scrutiny in many countries. Consequently, selective DCT strategies have been put in place to constrain the use of DCT only for treatment of chronically infected cows (Berry and Hillerton, 2002b; Pyörälä, 2002). In addition, internal teat sealants have also been used in lieu of or in combination with antimicrobial DCT to provide additional support for the physical barrier of the teat canal (TC) against transgression of mastitis pathogens (Berry and Hillerton, 2002a; Bhutto et al., 2011). However, despite implementation of the abovementioned intervention strategies, peripartal mastitis still remains a major problem worldwide (Pyörälä, 2008; De Vliegher et al., 2012). This notion underscores 
the necessity of thorough evaluation of the effectiveness of common DCT strategies in controlling mastitis pathogens. Moreover, it is also important to evaluate the modulatory effect of common DCT strategies on the microbiota of different niches of the udder. Within microbial ecosystems, particularly those associated with eukaryotic hosts, bacteria do not live in isolation but rather co-exist in complex communities where alteration in the proportion of one species may change the functionality of the community as a whole and the way it influences the physiology of the host (Faust and Raes, 2012).

To date, a combination of culture-dependent and DNA-based techniques have been employed to explore the diversity of microbial communities that colonize the teat apex (Woodward et al., 1987; Braem et al., 2012; Verdier-Metz et al., 2012) and TC (Falentin et al., 2016) of dairy cows. Moreover, a growing body of evidence suggests that in addition to recognized mastitis pathogens, which usually possess a variety of virulence factors enabling them to resist the defense mechanisms of the udder (Barkema et al., 2006; Melchior et al., 2006), a diverse range of opportunistic and commensal bacteria can inhabit colostrum (Lima et al., 2017) and milk of healthy cows (Kuehn et al., 2013; Oikonomou et al., 2014; Ganda et al., 2016). Phylogenetic relatedness among the microbiota of teat apex, TC, and mammary secretions suggest that a constant microbial exchange exist among these interconnected niches of the udder. This hypothesis is supported by the results of previous studies indicating that the microbial colonization of the TC with certain staphylococcal species can serve as an important reservoir for the development of new IMIs during lactation (Haveri et al., 2008; Taponen et al., 2008; Quirk et al., 2012). Comparative analyses of the microbiota of milk, teat skin, and bedding materials have also suggested that teat skin is the most important source of intramammary microbiota (Doyle et al., 2016; Fehr et al., 2017). Hence, to gain a better understanding of the effectiveness of common DCT strategies in controlling mastitis pathogens and modulating udder microbiota, peripartal dynamics of the microbiota of different niches of the udder should be evaluated simultaneously. Recently, it was demonstrated that the milk microbiota of a healthy udder quarter is highly resilient against DCT using ceftiofur hydrochloride and internal teat sealant (Bonsaglia et al., 2017). The aim of the present observational study was to concurrently evaluate shifts in the microbiota composition of TC and mammary secretions of healthy udder quarters following administration of antimicrobial DCT in combination with internal teat sealant.

\section{MATERIALS AND METHODS}

\section{Ethics Statement}

The research protocol of the present study was reviewed and approved by the Animal Care Committee of the University of Manitoba (protocol number F14-027).

\section{Selection of Animals and Sample Collection}

Eleven late-pregnant lactating Holstein cows were selected from a commercial dairy farm located in Manitoba, Canada, based on the following inclusion criteria: no history of clinical mastitis or antibiotic treatment in the 4 mo preceding drying off, 4 functional udder quarters with no (sub)clinical mastitis at drying off, no clotting or abnormal appearance of milk, California mastitis test score $=0$, absence of udder edema and redness, and quarter SCC $<200,000$ cells/mL to ensure absence of severe inflammatory responses in individual quarters (SCC analyses were performed at the Horizon Lab Ltd., Winnipeg, MB, Canada, and are presented in Supplemental Table S1; https://doi.org/10.3168/jds $.2018-14858)$. Cows were housed in freestall pens and milked 3 times a day at 0400, 1200, and $2000 \mathrm{~h}$, until the end of the lactation and were dried off abruptly. At drying off, all of the quarters of all cows were subjected to blanket DCT by administration of a long-acting antimicrobial product containing 200,000 units of penicillin $\mathrm{G}$ and $400 \mathrm{mg}$ of novobiocin per 10-mL tube (NovaDry, Zoetis, Kirkland, QC, Canada) in combination with internal teat sealant (Orbeseal, Zoetis). Selected animals gradually entered the sampling procedure of this study during October 2014 to March 2015. Aseptic TC swabs and corresponding milk and colostrum samples were collected from all quarters of all cows once at drying off and a second time immediately after calving. Average duration of dry period was $58.77 \mathrm{~d}( \pm 8.35 \mathrm{~d})$. The TC swab samples were collected using Ultrafine HydraFlock fiber-tipped swabs (Puritan, Guilford, ME). Prior to sampling, teat ends were thoroughly scrubbed with cotton pads moistened in $70 \%$ isopropyl alcohol. The swab was inserted $\sim 5 \mathrm{~mm}$ into the distal end of the TC and rotated $360^{\circ}$. The swab tip was then placed into a sterile transport tube containing $1 \mathrm{~mL}$ of Liquid Amies sterile medium (Puritan) and aseptically broken at its breakpoint by pressing against the bottom of the transport tube. This procedure was repeated to obtain a second swab sample from each teat end. Transport tubes were then placed on ice until transfer to laboratory and storage at $-80^{\circ} \mathrm{C}$. Quarter milk samples were collected following standard recommendations of the 
National Mastitis Council: before sampling, 3 streams of foremilk were discarded to minimize chances of sample contamination from bacteria colonizing the TC. Pre-milking teat disinfection was performed using $0.5 \%$ iodine pre-dip solution, and teats were thoroughly dried using individual paper towels and then scrubbed for 15 s using cotton pads moistened in $70 \%$ isopropyl alcohol. Milk and colostrum samples $(\sim 15 \mathrm{~mL})$ were then collected into sterile containers and placed on ice until transfer to laboratory and aliquotting. Upon arrival at laboratory, from each sample, one aliquot of $1.5 \mathrm{~mL}$ was stored at $-80^{\circ} \mathrm{C}$ until processed for DNA extraction and microbial analysis and 2 backup aliquots of $4 \mathrm{~mL}$ and $\sim 8 \mathrm{~mL}$ were stored at -80 and $-20^{\circ} \mathrm{C}$, respectively.

\section{DNA Extraction from Swab, Milk, and Colostrum Samples}

Genomic DNA from TC swab samples was extracted using ZR-96 well Fungal/Bacterial DNA Kit (Zymo Research, Irvine, CA) following modified protocols of the manufacturer as follows: tubes containing swabs and transport medium were defrosted at $4^{\circ} \mathrm{C}$ for $4 \mathrm{~h}$, vortexed for $2 \mathrm{~min}$, and centrifuged at 12,000 $\times \mathrm{g}$ for 15 min. Supernatants were removed and pellets and swab tips were resuspended by adding $200 \mu \mathrm{L}$ of PBS buffer and vortexing for $30 \mathrm{~s}$. Next, $1 \mathrm{~g}$ of autoclaved $0.5 \mathrm{~mm}$ silica beads, $400 \mu \mathrm{L}$ of Lysis Solution (Zymo Research), and $18 \mu \mathrm{L}$ of $20 \mathrm{mg} / \mathrm{mL}$ Proteinase K (Zymo Research) were added to each tube, vortexed for 2 min using a 2010 GenoGrinder (SPEX SamplePrep, Metuchen, NJ) at 1,700 strokes per min, and subsequently incubated in a heated shaker at $45^{\circ} \mathrm{C}$ for $45 \mathrm{~min}$. Four hundred microliters of the resulting mixture was then transferred to the deep-well plate of the Fungal/Bacterial DNA Kit and the extraction process continued following the manufacturer's protocol (https://www.zymoresearch .eu/quick-dna-fungal-bacterial-kit). Milk samples were processed as follows: $1.5 \mathrm{~mL}$ of milk samples were centrifuged at $12,000 \times g$ for $20 \mathrm{~min}$ at $4^{\circ} \mathrm{C}$. Supernatants were carefully removed and $200 \mu \mathrm{L}$ of Tris-EDTA buffer and $300 \mu \mathrm{L}$ of $0.5 M$ EDTA $(\mathrm{pH}=8)$ were added to the pellet. The mixture was incubated for $20 \mathrm{~min}$ at room temperature and again centrifuged at 12,000 $\times g$ for $10 \mathrm{~min}$ at $4^{\circ} \mathrm{C}$. Supernatants were removed and pellets were resuspended by adding $200 \mu \mathrm{L}$ of PBS buffer and vortexing for $30 \mathrm{~s}$. Genomic DNA extraction from the resuspended pellets was then continued similar to the protocol described for swab samples. Negative controls $(\mathrm{n}=3)$ were included in both swab (using sterile swabs and transportation medium) and milk (using 1 $\mathrm{mL}$ of DNA-free sterile water; Thermo Fisher Scientific, Burlington, ON, Canada) extraction protocols.

\section{PCR Amplification and Construction of Sequencing Libraries}

The PCR was targeted to amplify V1-V2 regions of the bacterial 16S rRNA genes using modified F27/R357 primers (see Supplemental Table S2, https://doi.org/10 $.3168 /$ jds.2018-14858, for the complete list of primers used for PCR amplification and sequencing reactions). The forward PCR primer was indexed with 12-base Golay barcodes, allowing for multiplexing of samples. For each sample, PCR reaction was performed in duplicate and contained $3.0 \mu \mathrm{L}$ of extracted genomic DNA, 1.0 $\mu \mathrm{L}$ of each forward and reverse primer $(5 \mu M), 0.4 \mu \mathrm{L}$ of $20 \mathrm{mg} / \mathrm{mL}$ of BSA (Thermo Fisher Scientific), 11.6 $\mu \mathrm{L}$ of nuclease-free water (Thermo Fisher Scientific), and $10 \mu \mathrm{L}$ of 5 Prime Hot MasterMix (5 Prime Inc., Gaithersburg, MD). Reactions consisted of an initial denaturing step at $94^{\circ} \mathrm{C}$ for $3 \mathrm{~min}$ followed by $32 \mathrm{am}-$ plification cycles at $94^{\circ} \mathrm{C}$ for $30 \mathrm{~s}, 55^{\circ} \mathrm{C}$ for $20 \mathrm{~s}$, and $72^{\circ} \mathrm{C}$ for $20 \mathrm{~s}$, with a final extension step at $72^{\circ} \mathrm{C}$ for 5 min in an Eppendorf Mastercycler pro (Eppendorf, Hamburg, Germany). The sequencing library was then generated as described (Derakhshani et al., 2016) and sequenced using a MiSeq Reagent Kit V3 (600-cycle, Illumina, San Diego, CA) at the Gut Microbiome and Large Animal Biosecurity Laboratories, Department of Animal Science, University of Manitoba, Winnipeg, $\mathrm{MB}$, Canada. The sequencing data were deposited into the Sequence Read Archive (SRA) of National Center for Biotechnology Information (http://www.ncbi .nlm.nih.gov/sra) and can be accessed via accession numbers SRR7267363-SRR7267478. Metadata used to process sequences and perform statistical analyses are presented in Supplemental Table S1 (https://doi.org/ 10.3168/jds.2018-14858).

\section{Bioinformatics and Statistical Analyses}

The default settings of FLASH assembler (version 1.2.11; Magoc and Salzberg, 2011) were used to merge the overlapping paired-end Illumina fastq files. The UPARSE algorithm (Edgar, 2013) was used for (a) quality filtering of the reads based on maximum expected error value $=1.0$, (b) identification of unique sequences, (c) abundance sorting and removal of singletons, (d) clustering the reads into operational taxonomic units (OTU) based on 97\% identity threshold, (e) de novo and reference-based chimera checking (against GOLD database [20]), and (f) construction of an OTU table. Taxonomic classification was then carried out using QIIME (Caporaso et al., 2010b) implementation of UCLUST (version $=1.2 .22$; Edgar, 2010) and aligned against the Greengenes database (release May 2013) 
using the PyNAST algorithm (Caporaso et al., 2010a). Due to the importance of staphylococci and streptococci species to udder health and pathogenesis of mastitis, sequences of representative OTU within these 2 genera were further assigned taxonomy at the species level based on top BLASTN bit-scores with minimum sequence identities of $98 \%$ to representative sequences deposited in the National Center for Biotechnology Information-16S rRNA gene database.

The resulting OTU table was filtered to remove all the samples with low sequencing depths $(<4,500$ sequences per sample). Consequently, samples belonging to the cows that were left with $<2$ TC and corresponding milk samples were also removed from downstream analyses. Community richness (Chao1 estimate of species richness) and diversity (Shannon and Simpson's indices of diversity) were calculated using QIIME default scripts at an even depth of 4,500 sequences per sample. $\beta$-diversity metrics were calculated based on Bray-Curtis dissimilarities of the microbial communities following cumulative sum scaling (Paulson et al., 2013) normalization of the final OTU table. Principal coordinate analysis was applied on the resulting distance matrices to generate 2-dimensional ordination plots.

Normality of residuals for $\alpha$-diversity measurements was tested using the UNIVARIATE procedure of SAS (SAS 9.3, SAS Institute Inc., Cary, NC). Nonnormally distributed data were either log or Box-Cox power transformed and then subjected to ANOVA test using PROC MIXED of SAS. All pairwise comparisons among groups were tested using the Tukey studentized range adjustment. Permutational multivariate ANOVA (PERMANOVA; implemented in Primer6 software) was used to detect differences between $\beta$-diversity metrics of microbial communities. Label permutations (n $=9,999)$ were used in PERMANOVA to estimate the distribution of test statistics under the null hypothesis that within-group Bray-Curtis measures are not significantly different from between-group ones. The effect of cow was treated as a random factor in both ANOVA and PERMANOVA comparisons.

Proportions of bacterial taxa in pre-DCT versus postpartum microbiota were compared using multivariate analysis with linear modeling (Morgan et al., 2012) following filtering low prevalent OTU from the OTU table (OTU with less than 10 counts and presented in less than 2 samples were removed). In this approach, potential confounding factors, including different niches of the udder (milk and colostrum vs. TC), lactation stage (pre-DCT vs. postpartum), time of sampling, and individual animals (treated as a random factor), were fitted in a multivariate linear model for exploring their associations with the relative abundances of features (OTU, genera, or phyla). To avoid over-parameterization, the initial model was then boosted independently for each feature and only factors were kept in the final models that showed association with selected features. These factors were then used as predictors of the response variables (i.e., arcsin-square root transformed proportion of features). $P$-values were subjected to multiple hypothesis tests based on Benjamini and Hochberg false discovery rate (FDR), with significant associations being considered at a $q$-value (indicative of proportion of significant results that are false positives) $<0.10$.

Finally, the relationships among abundant bacterial genera ( $>1 \%$ of the community) and biodiversity metrics of the microbiota within each niche of the udder were explored using nonparametric Spearman's rank correlation coefficient test implemented in PAST (Hammer et al., 2012). The resulting correlation matrix was then visualized in heatmap using the corrplot package of R (Wei, 2017). All pairwise correlations with a $P$-value $<0.05$ were considered significant.

\section{RESULTS}

\section{Diversity of Udder Microbiota}

After quality filtering of the raw sequencing data, sequences belonging to $58 \mathrm{TC}$ swab samples and their corresponding milk and colostrum samples from 9 dairy cows were kept for downstream statistical analyses. Average sequencing depths for TC and mammary secretions were 44,314 $( \pm 18,301)$ and 20,709 $( \pm 10,821)$ sequences per sample, respectively. Sequencing output for negative controls was not considerable $(<100$ sequences per sample) and therefore excluded from downstream analyses. De novo clustering of sequences at $97 \%$ similarity threshold resulted in identification of $1,016( \pm 186)$ and $440( \pm 280)$ representative bacterial OTU for TC and mammary secretion, respectively. Comparison of diversity metrics between the microbiota of different niches of the udder indicated that the microbiota of TC samples were in general more species-rich (Chao1, $P<0.001$ ) and diverse (Shannon, $P<0.001$, and Simpson, $P<0.05)$ compared with the microbiota of their corresponding milk and colostrum samples (Figures $1 \mathrm{a}-\mathrm{c}$ ). Within niche comparisons of pre-DCT versus postpartum microbiota revealed no difference between the richness and diversity indices of the 2 sampling time points, with the exception of Chao1 estimates of species richness, which were higher $(P=$ 0.02 ) in pre-DCT milk microbiota than postpartum colostrum microbiota (Figures 1a-c). Supplemental Table 
S1 (https://doi.org/10.3168/jds.2018-14858) provides detailed information regarding diversity metrics and total number of representative OTU detected in each sample.

With regard to the $\beta$-diversity of microbial communities, principal coordinate analysis and PERMANOVA of Bray-Curtis dissimilarities revealed that the microbiota of TC samples were compositionally distinct from the microbiota of their corresponding milk and colostrum samples $\left(P_{\text {(PERMANOVA }}=0.0001 ;\right.$ Figure $\left.2 \mathrm{a}\right)$. In addition, in both niches of the udder, the pre-DCT microbiota was compositionally different than postpartum microbiota $\left(P_{\text {(PERMANOVA })}=0.002\right.$ and $P_{(\text {PERMANOVA })}=$ 0.001 for TC and mammary secretions, respectively). On the other hand, within both niches of the udder, the microbiota of quarters of the same cow were more similar to each other than to quarters of other cows $\left(P_{(\text {Permanova })}=0.0001\right)$, indicating the individuality of quarter microbiota (Figures $2 \mathrm{~b}$ and $2 \mathrm{c}$ ).

\section{Composition of Udder Microbiota}

Firmicutes, Proteobacteria, Bacteroidetes, and Actinobacteria were predominant bacterial phyla in the microbiota of both niches of the udder. Within the TC microbiota, DCT was associated with enrichment (FDR adjusted $q=0.065$ ) of the phylum Bacteroidetes, which constituted 15.2 and $19.1 \%$ of pre-DCT and postpartum microbiota, respectively (Figure 3a). Shifts were more pronounced in the microbiota of mammary secretions where a trade-off occurred between the proportions of Firmicutes and Proteobacteria (Figure 3b). The proportion of Firmicutes was higher (FDR adjusted $q=0.060$ ) in the microbiota of pre-DCT milk samples compared with postpartum colostrum samples (44.9 and 33.5\%, respectively), whereas the proportion of Proteobacteria was higher (FDR adjusted $q=0.059$ ) in the microbiota of postpartum colostrum samples than the microbiota of pre-DCT milk samples (44.7 and $32.1 \%$, respectively).

Figures $4 \mathrm{a}$ and $4 \mathrm{~b}$ show the proportions of abundant bacterial genera ( $>1 \%$ of the community) within the microbiota of TC and mammary secretions. Within the TC microbiota, several genera belonging to phyla Bacteroidetes (including unclassified Rikenellaceae, unclassified Bacteroidales, and 5-7N15) and Proteobacteria (including Pseudomonas) were enriched postpartum (FDR adjusted $q<0.10$ ), whereas the proportion of unclassified Bacillales (phylum Firmicutes) was higher in the pre-DCT microbiota. Proportions of other abundant genera of TC microbiota, including Corynebacterium, Staphylococcus, and unclassified Ruminococcaceae, did not change following drying off with application of DCT and internal teat sealant. On the other hand, comparison of pre-DCT versus postpartum microbiota
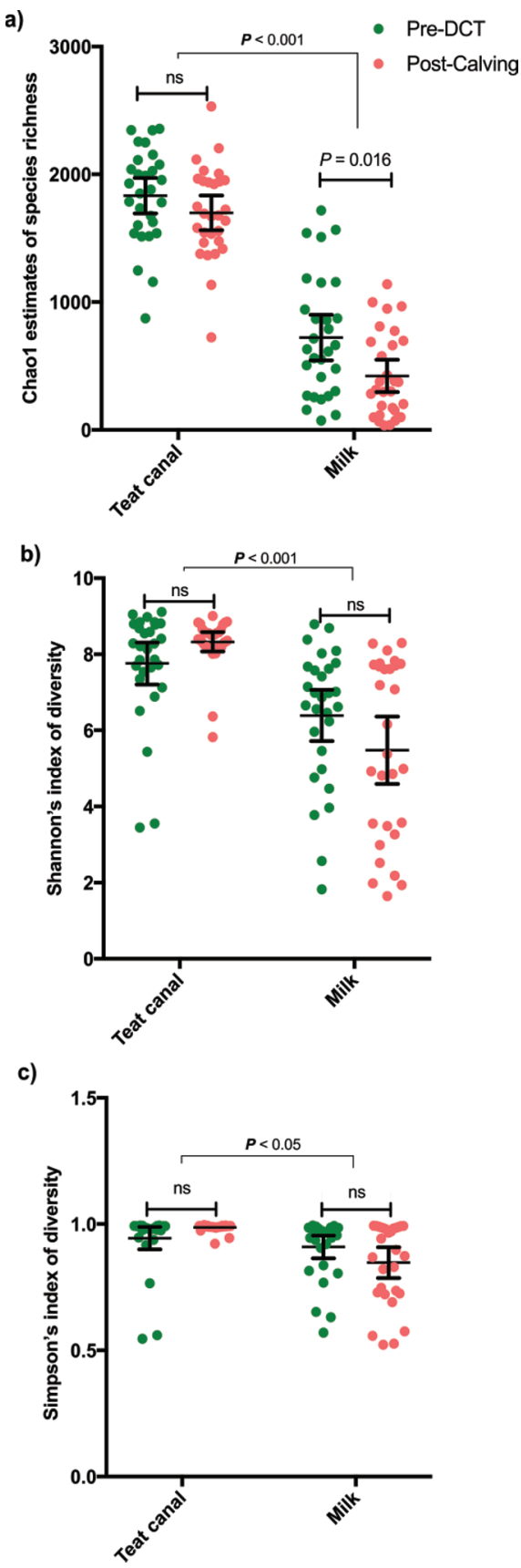

Figure 1. Shifts in the biodiversity metrics of teat canal and mammary secretions comparing pre-dry cow therapy (DCT) and postpartum. Alpha-diversity metrics including (a) Chao1 index of species richness, (b) Shannon's index of diversity, and (c) Simpson's index of diversity were calculated for teat canal and their corresponding mammary secretions (including milk and colostrum samples). Operational taxonomic unit (OTU) tables were normalized to an even depth of 4,500 OTU per sample before calculation of diversity metrics. Analysis of variance was performed to compare the mean of diversity metrics between the sampling time points (pre-DCT: samples collected at the time of drying off and before administration of dry cow therapy; postpartum: samples collected immediately after calving). A repeated measurement model was used to include the effect of individual cows as a random factor. Error bars denote the $95 \%$ CI. For all tests, $P$-values $<0.05$ were considered significant. ns indicates $P$-values $>0.05$. Color version available online. 

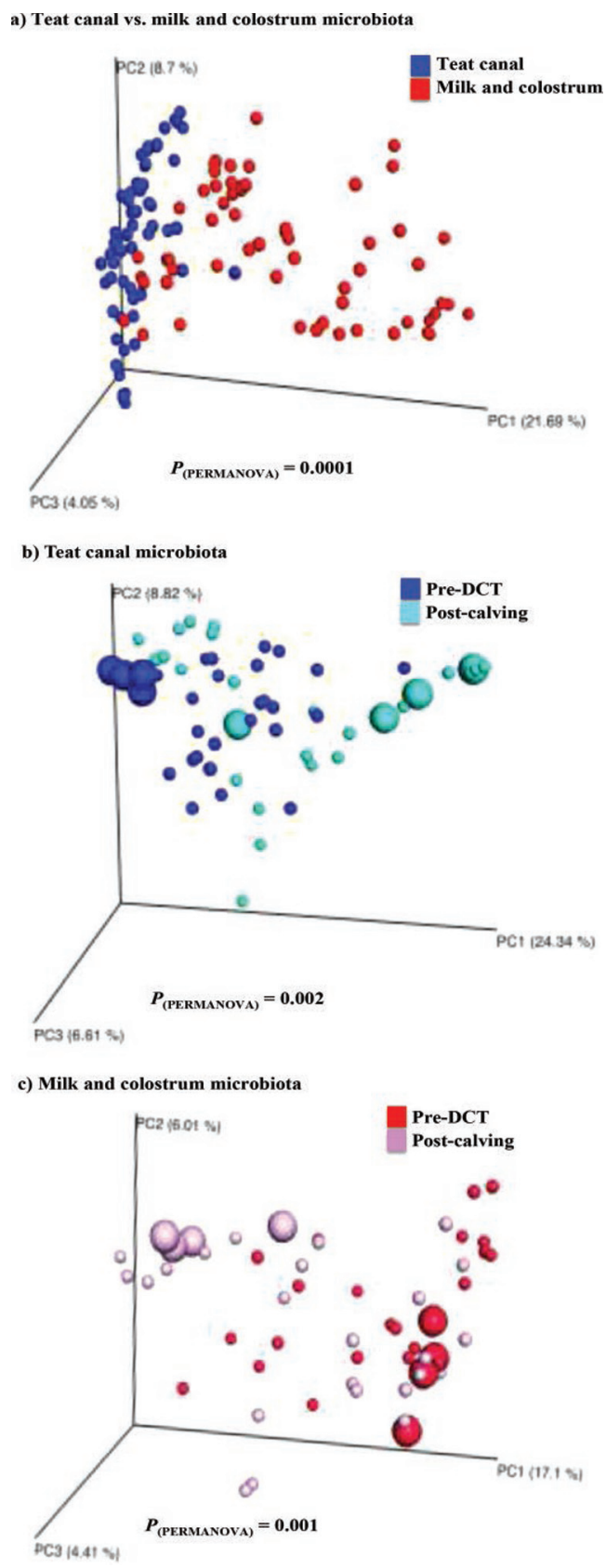

Figure 2. Beta-diversity of the bacterial communities of teat canal and mammary secretions. Principal coordinate (PC) analysis was used for visualization of Bray-Curtis dissimilarities of bacterial communities of (a) teat canal swab samples versus corresponding milk and colostrum samples, (b) teat canal swab samples collected at the time of drying off [pre-dry cow therapy (DCT)] versus immediately after parturition (postpartum), and (c) pre-DCT milk samples versus postpartum colostrum samples. Prior to calculation of Bray-Curtis dissimilarities, the operational taxonomic unit table was normalized using cumulative sum scaling transformation. Permutational multivariate ANOVA (PERMANOVA; 9,999 permutations) was performed using a repeated measurement model that included the effect of individual cows as a random factor. In panels b and c, pre-DCT and postpartum samples belonging to udder quarters of an individual animal have been enlarged to underscore the effect of individuality on the composition of udder microbiota. For all tests, $P$-values $<0.05$ were considered significant. Color version available online. of mammary secretions revealed that the proportion of several proteobacterial genera, including Pseudomonas, Stenotrophomonas, and unclassified Alcaligenaceae were enriched after calving (FDR adjusted $q<0.10$ ), whereas the proportion of genera belonging to Firmicutes (including Butyrivibrio and unclassified Clostridiales), and Actinobacteria (including Corynebacterium) were higher in the microbiota of pre-DCT milk samples.

\section{Associations of Abundant Bacterial Genera With Biodiversity of Udder}

Based on Spearman's rank correlation coefficient test, proportion of several bacterial genera was positively $(r h o \geq 0.5 ; P<0.05)$ or negatively $(r h o \leq-0.5$; $P<0.05)$ correlated with diversity metrics of the udder ecosystem (Figures $5 \mathrm{a}$ and $5 \mathrm{~b}$ ). Within the TC microbiota, the proportions of Corynebacterium and Staphylococcus were negatively correlated with Shannon and Simpson indices of diversity, whereas unclassified Ruminococcaceae, Lachnospiraceae, Clostridiales, and Bacteroidales were all positively correlated with richness (Chao1) and diversity (Shannon and Simpson) indices of the TC microbiota. Within the microbiota of mammary secretions, proportions of Mycobacterium, Stenotrophomonas, and unclassified Alcaligenaceae were negatively correlated with all indices of $\alpha$ - and $\beta$-diversity, whereas Clostridium, Butyrivibrio, unclassified Ruminococcaceae, Lachnospiraceae, and Clostridiales were positively correlated with biodiversity metrics of the milk and colostrum samples. Lactic acid bacteria, including Bifidobacterium and Lactococcus, were also positively correlated with microbiota richness and diversity of mammary secretions.

\section{Qualitative Comparison of Pre-DCT and Postpartum Microbiota}

Venn diagrams (Figures 6a-6d) were used to qualitatively compare the common genera and OTU between the microbiota of pre-DCT and postpartum time points. For the purpose of this analysis, we only used nonrandom genera/OTU that were present in at least $25 \%$ of the samples within each niche of the udder at each sampling time point. Within the TC microbiota, 78.5\% of bacterial genera and $63.7 \%$ of bacterial OTU were present at both sampling time points. On the other hand, the effect of drying off and DCT was more pronounced on the bacterial composition of the mammary secretions where 59.1 and $44.3 \%$ of bacterial genera and OTU were common between pre-DCT milk and postpartum colostrum samples, respectively. Qualitative comparison of common pathogenic or opportunistic bacterial groups (or both) revealed that within the TC 


\section{a) Teat canal microbiota}

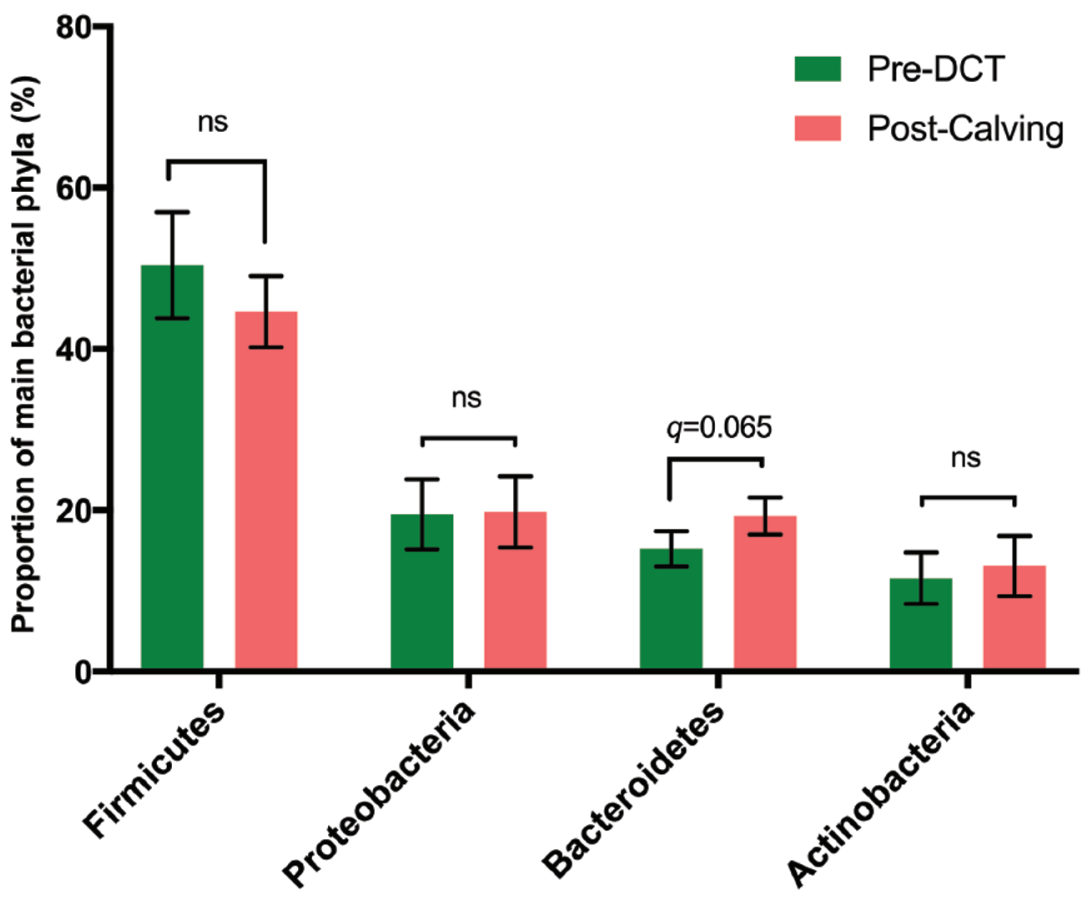

b) Milk and colostrum microbiota

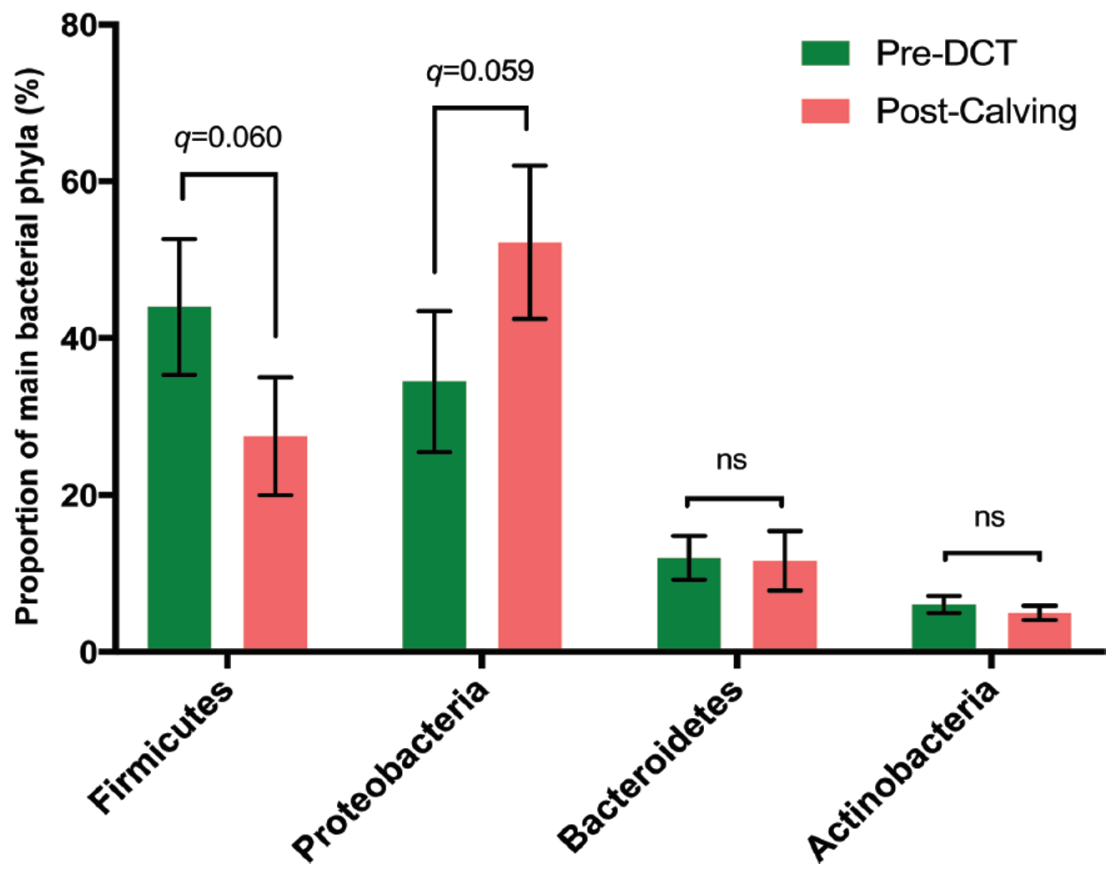

Figure 3. Shifts in the proportions of main bacterial phyla within the microbiota of teat canal and intramammary secretion comparing predry cow therapy (DCT) and postpartum. Bar graphs show the proportion of predominant bacterial phyla within the microbiota of (a) teat canal swab samples, and (b) corresponding milk and colostrum samples. Error bars denote the 95\% CI. Multivariate analysis with linear modeling was used to determine statistically significant differences among the relative abundances of bacterial phyla between the sampling time points. Multivariate analysis with linear modeling included a repeated measurement model including the effect of individual cows as random factor, sampling time point as predictor, and arcsin-square root transformed abundances of bacterial phyla as the response. Multiple hypotheses were adjusted by Benjamini and Hochberg false discovery rate, and significant associations were considered below a $q$-value (indicative of proportion of significant results that are false positives) threshold of 0.10 . ns indicates $q$-values $>0.10$. Color version available online. 


\section{a) Teat canal microbiota}

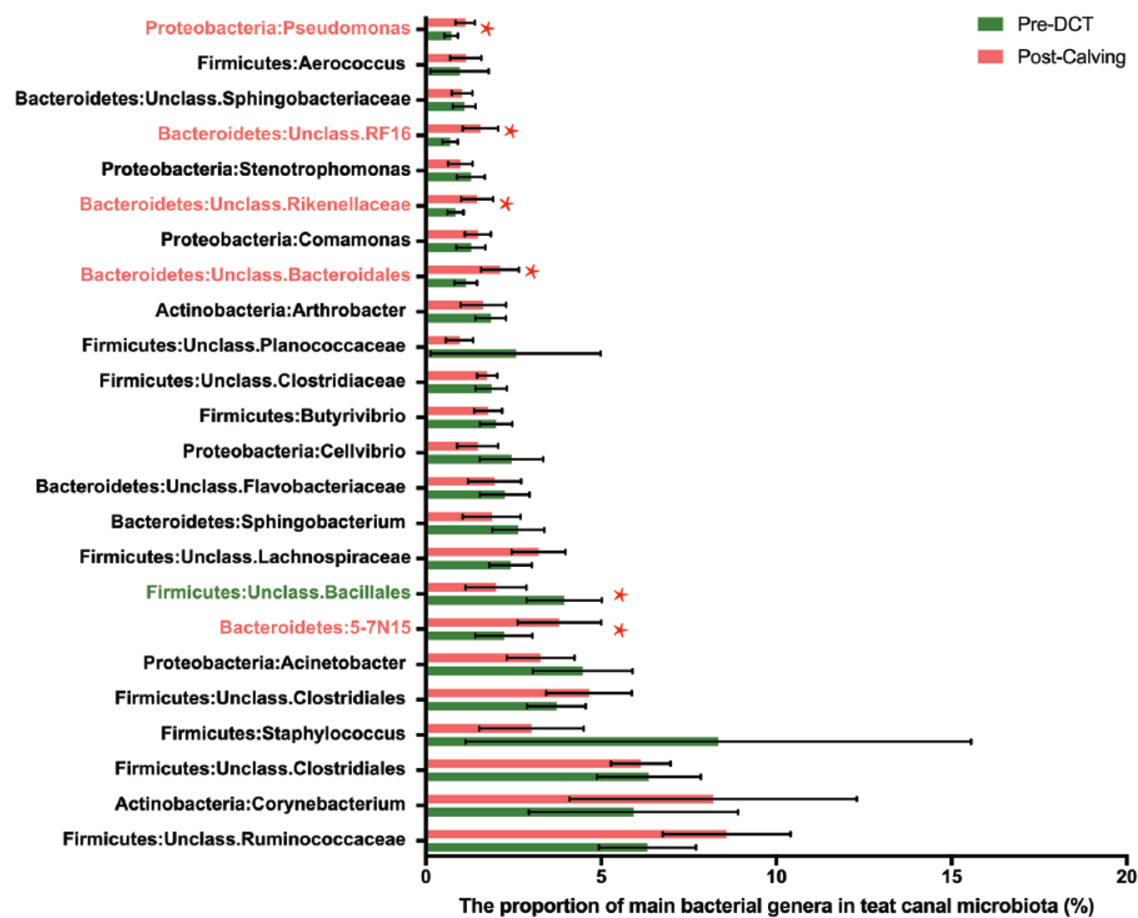

b) Milk/colostrum microbiota

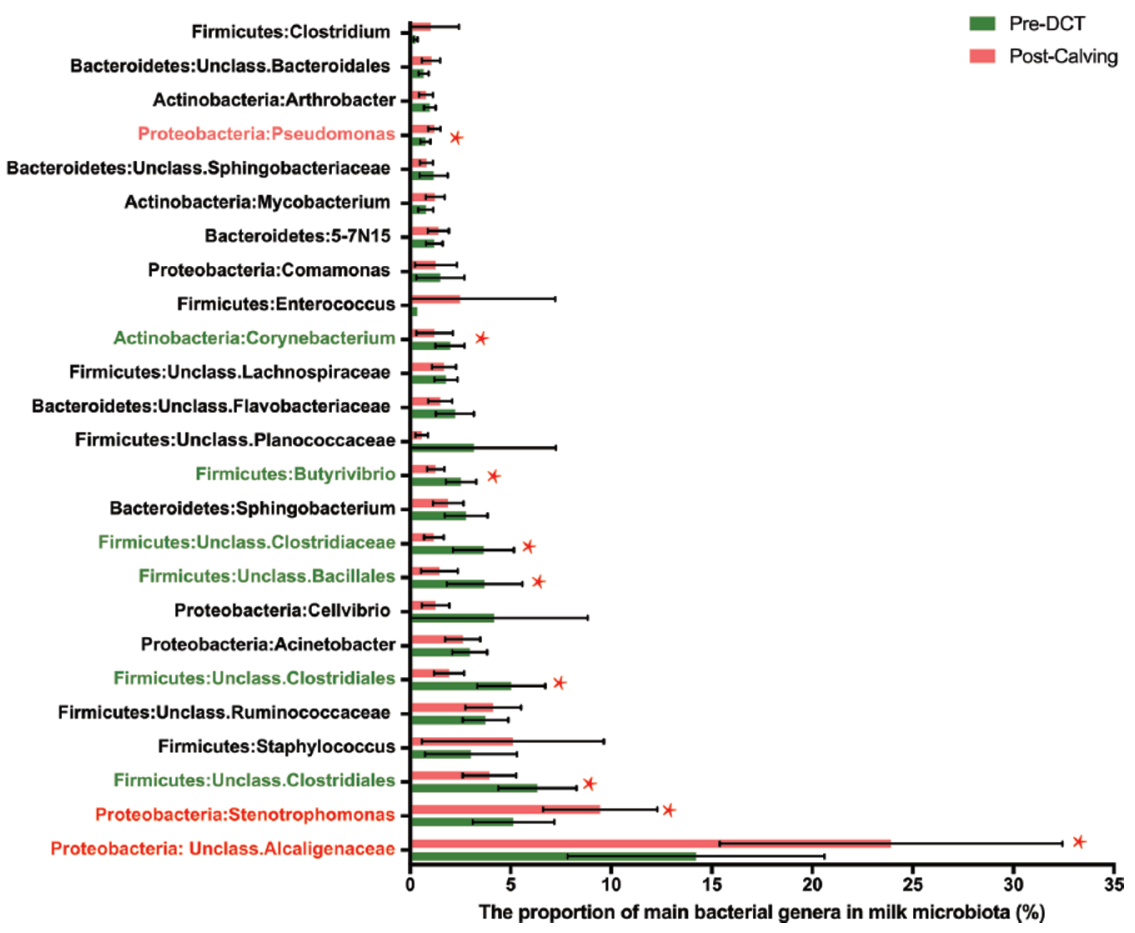

Figure 4. Shifts in the proportions of main bacterial genera within the microbiota of teat canal and intramammary secretion comparing pre-dry cow therapy (DCT) and postpartum. Bar graphs show the proportion of dominant bacterial genera (>1\% of the community) within the microbiota of (a) teat canal swab samples, and (b) corresponding milk and colostrum samples. Error bars denote the 95\% CI. Multivariate analysis with linear modeling was used to determine statistically significant differences among the relative abundances of bacterial genera between the sampling time points. Multivariate analysis with linear modeling included a repeated measurement model including the effect of individual cows as random factor, sampling time point as predictor and arcsin-square root transformed relative abundances of bacterial genera as the response. Multiple hypotheses were adjusted by Benjamini and Hochberg false discovery rate and significant associations were considered below a $q$-value (indicative of proportion of significant results that are false positives) threshold of 0.10 . *Asterisks indicate $q$-value $<0.10$. Color version available online. 
microbiota, genera Streptococcus, Staphylococcus, Corynebacterium, Campylobacter, unclassified Enterobacteriaceae, Pseudomonas, and Acinetobacter were part of the common microbiota between pre-DCT and postpartum sampling time points. All aforementioned genera, with the exception of Streptococcus and Campylobacter, were also identified as part of the common microbiota of pre-DCT and postpartum mammary secretions.

Additionally, due to the importance of streptococcal and staphylococcal species to mastitis control programs, their representative OTU were qualitatively compared between pre-DCT and postpartum microbiota of both niches of the udder using Venn diagram (Figures $5 \mathrm{c}$ and 5d). In addition, referred OTU were assigned taxonomy at the species level based on top BLASTN bit-scores with a minimum sequence identity of $97 \%$ with representative sequences deposited in the National Center for Biotechnology Information-16S rRNA gene database. Overall, 5 unique streptococcal OTU were identified within the microbiota of TC samples of which OTU classified as Streptococcus dysgalactiae, Streptococcus lutetiensis, and Streptococcus pluranimalium were common between pre-DCT and postpartum. In contrast, Strep. thermophilus, as well as unclassified streptococcal OTU1192, were only detected pre-DCT. With respect to staphylococci OTU, those belonging to Staphylococcus aureus, Staphylococcus equorum, Staphylococcus xylosus, Staphylococcus chromogenes, Staphylococcus sciuri, Staphylococcus auricularis, and Staphylococcus haemolyticus were common between pre-DCT and postpartum TC microbiota. Staphylococcus simulans was only detected in the microbiota of postpartum TC samples. On the other hand, in general, fewer streptococcal and staphylococcal OTU were identified within the microbiota of mammary secretions. Among these, streptococcal OTU, including those classified as Strep. dysgalactiae and Strep. lutetiensis, were exclusively detected in the microbiota of pre-DCT milk samples, whereas the majority of staphylococcal OTU, including those classified as Staph. aureus, Staph. xylosus, Staph.

\section{a) Teat canal microbiota}

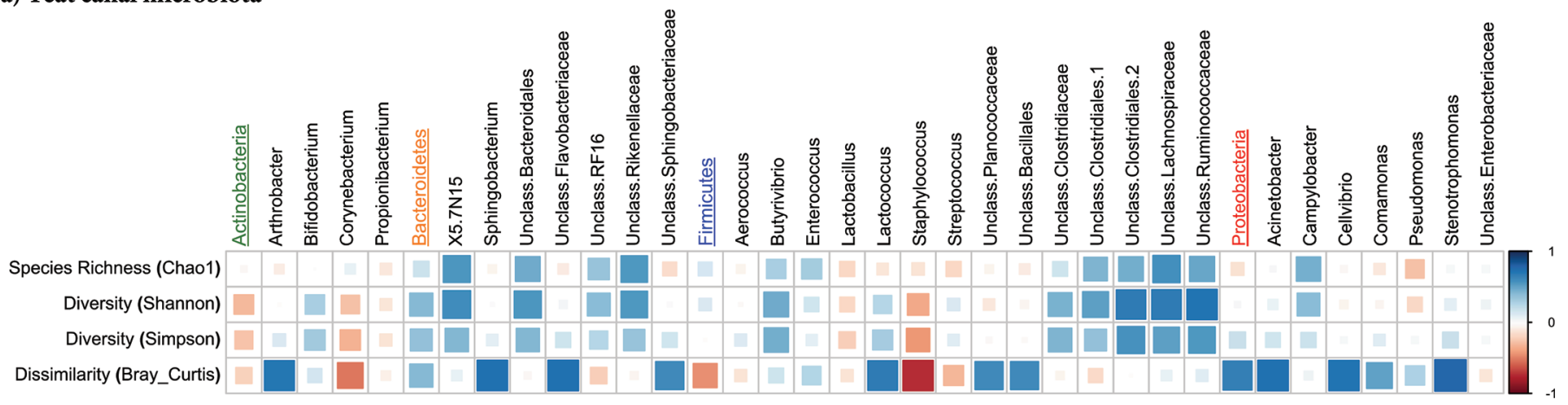

b) Milk and colostrum microbiota

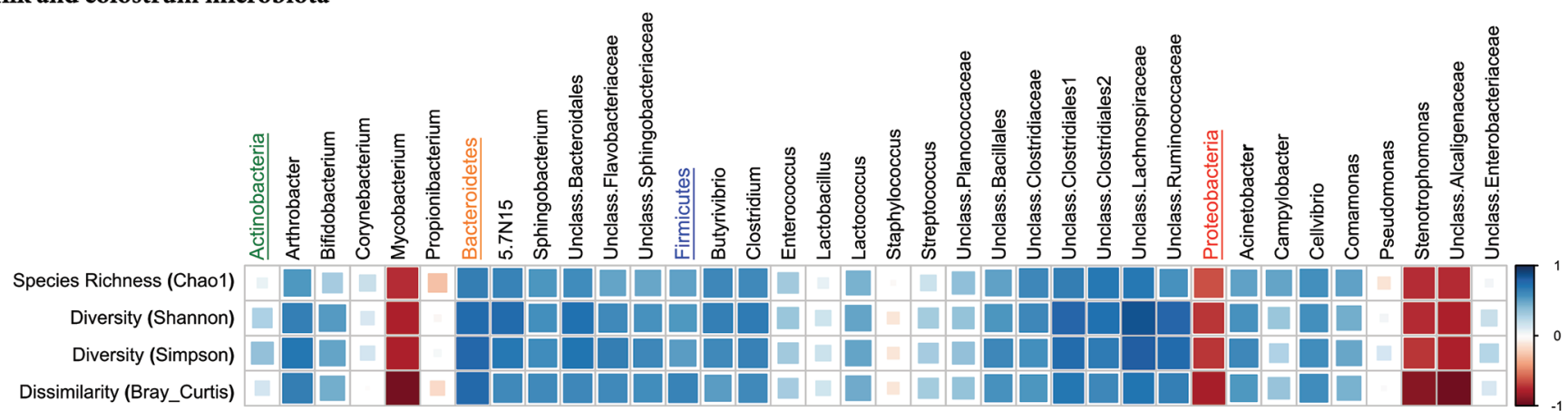

Figure 5. Niche-specific correlations between the proportion of abundant bacterial genera and diversity metrics within teat canal and intramammary secretion microbiota. The correlation matrix was based on the Spearman's rank correlation coefficient of the abundant bacterial genera ( $>0.1 \%$ of community) with diversity metrics within (a) teat canal microbiota and (b) intramammary microbiota. The strength of the correlation between each pair of variables is indicated by diameter and color of the squares. Black color shows a positive correlation, and white color shows a negative correlation. Color version is available online. In this case, the strength of coloration also shown by the color intensity. A color code of dark blue indicates a positive correlation coefficient close to +1 , and a color code of dark red indicates a negative correlation coefficient close to -1 . 
chromogenes, Staph. sciuri, and Staph. auricularis, were common between pre-DCT and postpartum microbiota of mammary secretions.

\section{DISCUSSION}

Although the efficacy of common DCT strategies in eliminating major mastitis pathogens that can be identified using bacteriological culture has been extensively investigated (Halasa et al., 2009; Petzer et al., 2009; Bradley et al., 2010, 2011), little is known about the dynamics of the native microbial communities of the udder during the dry period under the influence of blanket DCT. In the present study, we sought to address this knowledge gap by characterizing the dynamics of the microbiota inhabiting different ecological niches of the udder pre-DCT and postpartum. Overall, our results indicate that despite natural variations in the microbiota profile of clinically healthy udder quarters, administration of antimicrobial DCT in combination with internal teat sealant could alter the microbiota profile of both TC and mammary secretions during the dry period. Notwithstanding, we observed a high degree of commonality between pre-DCT and postpartum microbiota of both TC and mammary secretions, suggesting that udder microbiota is in general highly resilient against exposure to long-acting antimicrobials during the dry period.

\section{Inter- and Intra-Animal Variability in the Composition of Udder Microbiota}

Cows selected for the purposes of present study were raised under a similar management strategy, housed in the same freestall barn, and subjected to the same treatment strategy at drying off. Nonetheless, substantial variations existed among the microbiota profile of their clinically healthy udder quarters. This observation is in agreement with previous reports (Oikonomou et al., 2014; Falentin et al., 2016; Ganda et al., 2017) indicating that regardless of the inflammatory status of the udder, milk microbiota profiles can vary greatly among a) Teat canal microbiota Genus level

Shared

- Streptococcus

- Campylobacter

- Staphylococcus

- Corynebacterizan

- Enterobacteriaceae

- Pseudomonas

- Acinetobacter

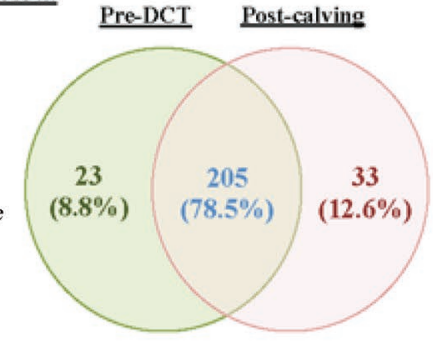

b) Milk microbiota Genus level

Pre-DCT

- Streptocaccus

- Campylobacter

Shared

- Staphylococcus

- Corynebacterim

- Enterobacteriaceae

- Pseudomonas

- Acinetobacter
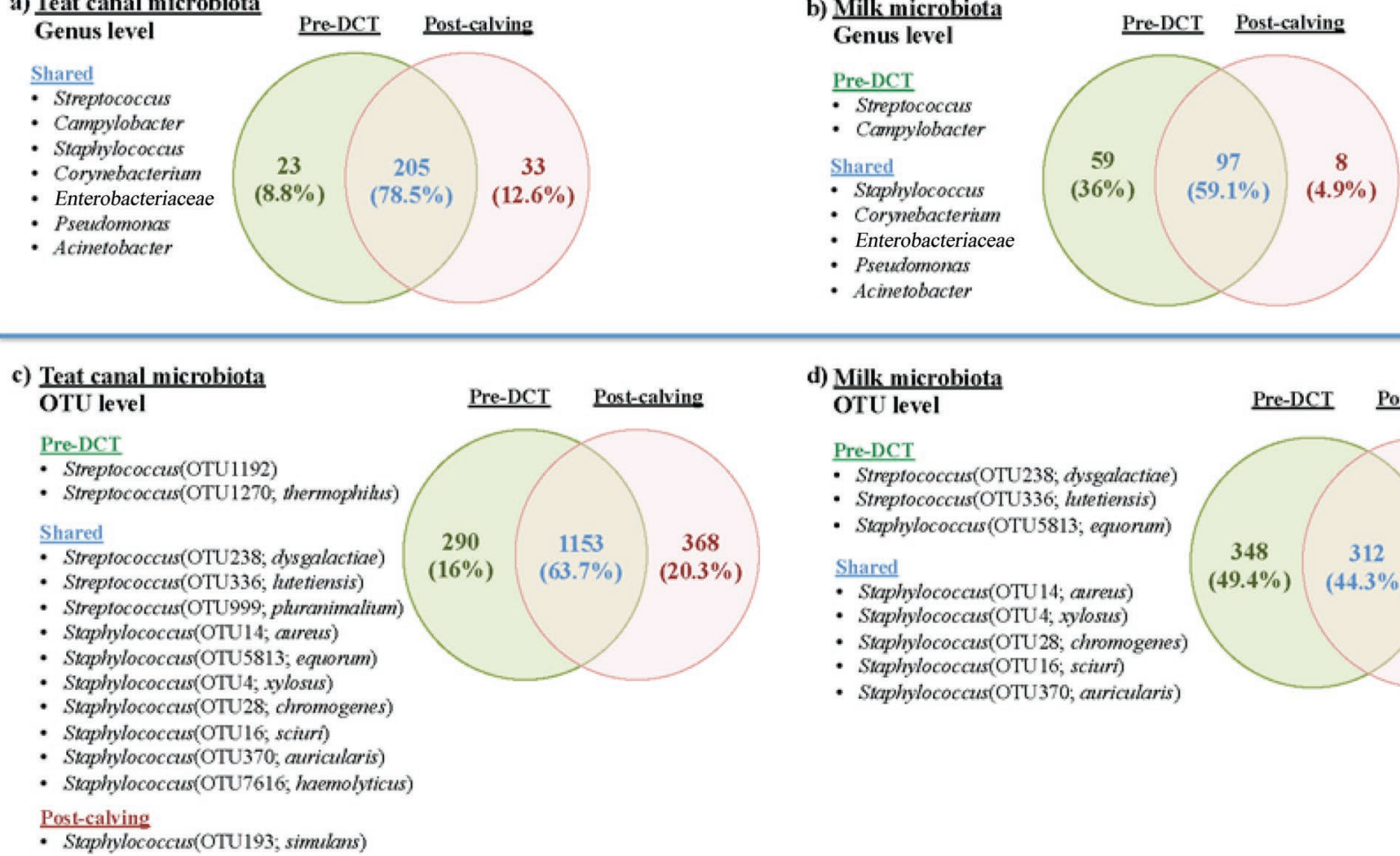

\section{d) Milk microbiota}

OTU level

Pre-DCT

- Streptococcus(OTU238; dysgalactiae)

- Streptococcus(OTU336; hutetiensis)

- Staphylococcus(OTU5813; equorum)

Shared

- Staphylococcus(OTU 14; careus)

- Staphylococcus(OTU4; xylosus)

- Staphylococcus(OTU28; chromogenes)

- Staphylococcus(OTU16; sciuri)

- Staphylococcus(OTU370; auricularis)

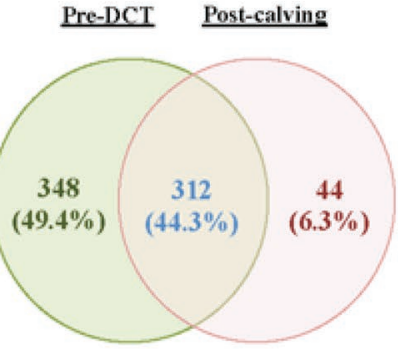

Figure 6. Qualitative comparison of the microbiota of teat canal and mammary secretions pre-dry cow therapy (DCT) and postpartum. Venn diagrams were used to qualitatively compare the presence/absence of common bacterial genera (a and b) and operational taxonomic units (OTU; $\mathrm{c}$ and d) between the nonrandom microbiota of teat canal and milk/colostrum samples collected at the time of drying off (pre-DCT) versus immediately after parturition (postpartum). Nonrandom microbiota was defined as the genera/OTU present in at least $25 \%$ of the samples within each niche of the udder at each sampling time point. The legend on the left of each diagram shows the presence/absence of certain bacterial lineages of interest (potential pathogenic/opportunistic groups). Color version available online. 
individual quarters. In the present study, in addition to the lack of clinical signs of mastitis, a SCC threshold of $<200,000$ cells $/ \mathrm{mL}$ was set as the inclusion criteria for udder quarters. However, despite the low SCC of preDCT milk samples, microbiota richness (Chao1 index) and total number of unique bacterial OTU detected in the microbiota of these samples varied greatly. The results suggest that microbiota richness cannot serve as an indicator of the inflammatory status of the udder. Similarly, we were unable to detect any association between Shannon and Simpson's indices of diversity and the SCC measurements of the pre-DCT milk samples (based on Spearman's rank correlation coefficient test). In contrast, others (Oikonomou et al., 2014; Ganda et al., 2016; Ganda et al., 2017) have reported that the dominance of certain mastitis pathogens, and consequently increased SCC, is associated with decreased diversity of the milk microbiota. In our previous effort to characterize the microbiota profiles of 144 quarters with a wide range of SCC (Derakhshani et al., 2017), we also found that SCC measurements of the quarter milk samples were negatively correlated with their Simpson's index of diversity. A plausible explanation for the inability of present study to find associations between biodiversity metrics and SCC may be the limited number of pre-DCT quarter milk samples $(\mathrm{n}=29)$ and their narrow range of SCC (from 13,000 to 174,000 cells $/ \mathrm{mL}$ with a median of 30,000 cells $/ \mathrm{mL}$ ). Notwithstanding, we believe that the indices of microbiota richness and diversity by themselves cannot be considered either sensitive or specific indicators of mastitis.

Setting aside natural variations in the composition of quarter microbiota, a high degree of individuality in the composition of quarter microbiota was present, meaning that the microbiota profile of quarters of the same cow were more similar to each other than udder quarters of other cows. This is in agreement with our previous findings (Derakhshani et al., 2017), but contradicts the results of Falentin et al. (2016), who did not observe inter-animal variability in the composition of quarter microbiota. We speculate that lower interanimal variability in the composition of quarter microbiota may be indicative of the synergetic influence of cows' genetic and physiological status on the microbiota of different niches of udder. In addition, the possibility that differences in the microbiota of cows' surrounding environments, such as bedding material, might have also contributed to the inter-animal difference in the composition of quarter microbiota cannot be ruled out, as the microbiota of bedding materials can be considered the primary source for microbial colonization of the teat skin.

\section{Shifts in Diversity and Composition of Udder Microbiota}

Overall, shifts were more pronounced within the microbiota of mammary secretions than the microbiota of TC samples. By comparing the composition of microbiota between pre-DCT milk and colostrum samples, dramatic changes occurred in the proportions of main bacterial phyla, Firmicutes and Proteobacteria, as well as their constituent genera and OTU. These findings are in contrast with the observations of Bonsaglia et al. (2017), who compared the microbiota profile of milk samples collected at the time of drying off (using internal teat sealant in combination with or without ceftiofur hydrochloride) and 1 wk postpartum. In the latter study, inclusion of ceftiofur hydrochloride was not associated with change in either species richness or diversity of the intramammary microbiota. In addition, proportions of main bacterial genera that are commonly regarded as potential mastitis pathogens, including Escherichia, Klebsiella, Mycoplasma, Staphylococcus, and Streptococcus, were also unchanged following DCT with ceftiofur hydrochloride. However, it is noteworthy to mention that postpartum milk samples in the referred study were collected 1 wk after calving and following exposure of the udder to environmental sources of bacteria. Therefore, the above-mentioned study remains inconclusive regarding the true effect that DCT has on the proportion of different bacterial groups throughout the dry period. Ganda et al. (2017) also reported that intramammary administration of ceftiofur hydrochloride in lactating cows was not associated with long-term changes in the composition of milk microbiota. By showing that the proportion of the 25 most abundant bacterial families was unchanged in response to antibiotic challenge, the authors of the latter study speculated that due to the very low bacterial load of the healthy milk, its microbial ecosystem is not competitive enough for favoring the overgrowth of certain bacterial lineages, in particular those that are resistant to antibiotic therapy. In contrast, in the present study, DCT using the combination of penicillin G and novobiocin was associated with decreased proportions of predominantly gram-positive Firmicutes on the one hand, and enrichment of gram-negative Proteobacteria on the other hand. Apart from influences that drying off and isolation of udder from environmental sources of microbiota, via administration of internal teat sealant, might have had on the microbiota of udder quarters in the present study, the above-mentioned controversial results can also be explained by differences in the spectra of the activity of antimicrobial compounds 
used in each study. Third-generation cephalosporins, including ceftiofur, have a broad-spectrum activity against both gram-positive and gram-negative bacteria (Hornish and Katarski, 2002). On the other hand, most DCT products, including the one selected for the purpose of the present study, have been traditionally chosen from antibiotics with narrower spectra of activity against gram-positive contagious pathogens such as Staph. aureus and Streptococcus agalactiae (Bradley et al., 2011). Here, we observed that proteobacterial genera, including Pseudomonas, Stenotrophomonas, and unclassified Alcaligenaceae, were enriched within the microbiota of postpartum colostrum samples. Interestingly, the latter 2 genera were also negatively correlated with all the indices of richness and diversity of intramammary microbiota. Microbiota dominance and decreased heterogeneity are proposed characteristics of mastitis-associated dysbiosis (Oikonomou et al., 2014). Stenotrophomonas spp., in particular S. maltophilia, have been occasionally isolated from mastitic quarters (Nam et al., 2009; Ohnishi et al., 2012). Predominance of Stenotrophomonas has been also reported within the milk microbiota of culture-negative mastitis samples (Kuehn et al., 2013). A plausible explanation for the overrepresentation of Stenotrophomonas within the microbiota of colostrum samples in the present study would be the well-documented ability of this bacterial lineage to resist a wide range of broad-spectrum antibiotics (Looney et al., 2009; Ohnishi et al., 2012). In another study investigating the effect of high-concentrate dietary challenge on the milk microbiota of primiparous Holstein dairy, Zhang et al. (2015) reported that the proportion of genera Stenotrophomonas and Alcaligenes was enriched within the milk microbiota of cows experiencing subacute ruminal acidosis, although the authors were not able to establish any link between the proportion of these genera and inflammatory status of the udder. Similar to Stenotrophomonas spp., Alcaligenes spp. are also nonfermentative gram-negative bacteria showing resistance against a wide range of broad-spectrum antibiotics (Tena et al., 2015). Despite sporadic reports on the isolation of strains from quarters with clinical mastitis in dairy cows (Petrovski et al., 2011) and humans (Patel et al., 2016), Alcaligenes spp. are in general considered opportunistic bacteria capable of causing nosocomial infections in immunocompromised patients (Tena et al., 2015). Taken together, our results suggest that post DCT state of the intramammary microbiota is in favor of overgrowth by opportunistic gram-negative bacteria, whereas on the other hand, a significant reduction was observed in the proportion of gram-positive commensals, including Butyrivibrio and Clostridiales, which have been reported as predominant colonizers of healthy milk microbiota (Addis et al., 2016). It is noteworthy to mention that shifts in the composition of udder microbiota in the dry period and around calving could also be partly attributed to the differences in the composition of metabolite and immune components of the milk and colostrum (Gopal and Gill, 2000; Stelwagen et al., 2009). Oligosaccharides and different classes of immunoglobulins are examples of bioactive compounds in mammary secretions capable of modulating the composition of intramammary microbiota (Addis et al., 2016; Aakko et al., 2017).

As stated earlier, compared with the microbiota of mammary secretions, a higher proportion of bacterial OTU was common between pre-DCT and postpartum TC microbiota. This suggests that although internal teat sealant can provide barrier against the entrance of new bacterial species into the udder, as evidenced by the overall reduction in total bacterial OTU detected postpartum, it may also limit the access of antimicrobial compounds to the internal lining of the TC and hence limit the effectiveness of DCT in eliminating chronic bacterial infections within this niche of the udder. Notably, the majority of staphylococcal OTU detected in the TC microbiota before drying off, including Staph. aureus and other non-aureus staphylococci such as Staph. xylosus, Staph. chromogenes, Staph. sciuri, and Staph. haemolyticus were also present in the postpartum TC microbiota. Earlier studies (Haveri et al., 2008; Taponen et al., 2008; Quirk et al., 2012) have indicated that colonization of the TC with staphylococcal species may serve as an important reservoir for development of IMI. In the present study, in addition to staphylococcal OTU, a wide range of bacterial OTU belonging to genera that are often associated with pathogenic or opportunistic characteristics (or both) within the udder ecosystem, including Corynebacterium, unclassified Enterobacteriaceae, and Pseudomonas, were also identified as common colonizers of the postpartum microbiota in both TC and colostrum samples. Lima et al. (2017) also reported that the colostrum microbiota of multiparous cows receiving blanket antimicrobial DCT using cloxacillin benzathine, which has a narrow-spectrum bactericidal activity against gram-positive bacteria, were composed of a high proportion of OTU belonging to genera Staphylococcus, unclassified Enterobacteriaceae, and Pseudomonas. Obviously, DNA-based profiling of the microbiota is not a sound approach for drawing conclusions with respect to the viability of different bacterial groups and persistency of their infections. Indeed, extracellular DNA that are either passively released from dead bacterial cells or actively secreted by living bacteria can persist in the tissue and biological fluids of the body for varying time periods (Thomas and Nielsen, 
2005; Nielsen et al., 2007), and therefore, may result in generation of false-positive signals through PCR amplification and $16 \mathrm{~S}$ rRNA gene sequencing. Nonetheless, our findings underscore the need for thorough evaluation of the effectiveness of current DCT strategies in eliminating mastitis pathogens capable of colonizing the TC, and to further assess their potential links with development of IMI during the dry period, subsequent milking, or both. Parallel metagenomic and metatranscriptomic investigations of the mammary microbiome can provide novel insights into the influence of DCT on the persistence and activity of different bacterial lineages, in particular, regarding expression and spread of antimicrobial resistance genes among pathogenic and opportunistic bacterial groups.

An unexpected finding of the present study was identification of OTU that were exclusively detected in the postpartum microbiota of either niche of the udder. Several quarter level factors, including teat end hyperkeratosis, integrity of the teat orifice, and formation of the keratin plug of the TC, have been associated with susceptibility to development of new IMI during the dry period (Dingwell et al., 2004). However, use of internal teat sealant at the time of drying off reduces the incidence rate of new IMI during the dry period or early lactation (Godden et al., 2003; Rabiee and Lean, 2013). In the present study, the total number of bacterial OTU that were exclusively detected in the colostrum microbiota was considerably lower than what was observed in the postpartum TC microbiota. Nevertheless, because the presence of an endogenous route of colonization has not yet been proven within the bovine udder, detection of new OTU within the microbiota of postpartum samples may be indicative of the inability of internal teat sealant to provide full protection against transgression of environmental bacteria past the TC, or perhaps it might be as a result of exogenous contaminations introduced during the intramammary injection of DCT compounds at the time of drying off.

\section{CONCLUSIONS}

Shifts were more pronounced within the microbiota of mammary secretions than the microbiota of TC. Reduction in the species richness of intramammary microbiota was accompanied by enrichment of proteobacterial genera. However, despite minor compositional differences, the qualitative analysis revealed a high degree of commonality between the microbiota of samples collected before drying off and postpartum. An important observation was that a high percentage of bacterial genera that are commonly regarded as mastitis pathogens were common between pre-DCT and postpartum microbiota of mammary secretions and TC samples. Collectively, these results suggest that the microbiota of different niches of the udder are highly resilient against exposure to long-acting antimicrobials during the dry period.

\section{ACKNOWLEDGMENTS}

The present research was supported by grants from Natural Science and Engineering Research Council of Canada - Discovery Program; Growing Forward 2-Agricultural Rural Development Initiative Program of the Province of Manitoba, Canada; and Dairy Farmers of Manitoba, Canada.

\section{REFERENCES}

Aakko, J., H. Kumar, S. Rautava, A. Wise, C. Autran, L. Bode, E. Isolauri, and S. Salminen. 2017. Human milk oligosaccharide categories define the microbiota composition in human colostrum. Benef. Microbes 8:563-567.

Addis, M. F., A. Tanca, S. Uzzau, G. Oikonomou, R. C. Bicalho, and P. Moroni. 2016. The bovine milk microbiota: Insights and perspectives from -omics studies. Mol. Biosyst. 12:2359-2372.

Barkema, H. W., Y. H. Schukken, and R. N. Zadoks. 2006. Invited review: The role of cow, pathogen, and treatment regimen in the therapeutic success of bovine Staphylococcus aureus mastitis. J. Dairy Sci. 89:1877-1895.

Berry, E. A., and J. E. Hillerton. 2002a. The effect of an intramammary teat seal on new intramammary infections. J. Dairy Sci. $85: 2512-2520$.

Berry, E. A., and J. E. Hillerton. 2002b. The effect of selective dry cow treatment on new intramammary infections. J. Dairy Sci. $85: 112-121$.

Bhutto, A. L., R. D. Murray, and Z. Woldehiwet. 2011. The effect of dry cow therapy and internal teat-sealant on intra-mammary infections during subsequent lactation. Res. Vet. Sci. 90:316-320.

Bonsaglia, E. C., M. S. Gomes, I. F. Canisso, Z. Zhou, S. F. Lima, V L. Rall, G. Oikonomou, R. C. Bicalho, and F. S. Lima. 2017. Milk microbiome and bacterial load following dry cow therapy without antibiotics in dairy cows with healthy mammary gland. Sci. Rep. $7: 8067$.

Bradley, A. J., J. E. Breen, B. Payne, and M. J. Green. 2011. A comparison of broad-spectrum and narrow-spectrum dry cow therapy used alone and in combination with a teat sealant. J. Dairy Sci. 94:692-704.

Bradley, A. J., J. E. Breen, B. Payne, P. Williams, and M. J. Green 2010. The use of a cephalonium containing dry cow therapy and an internal teat sealant, both alone and in combination. J. Dairy Sci. 93:1566-1577.

Bradley, A. J., and M. J. Green. 2004. The importance of the nonlactating period in the epidemiology of intramammary infection and strategies for prevention. Vet. Clin. North Am. Food Anim. Pract. 20:547-568.

Braem, G., S. De Vliegher, B. Verbist, M. Heyndrickx, F. Leroy, and L. De Vuyst. 2012. Culture-independent exploration of the teat apex microbiota of dairy cows reveals a wide bacterial species diversity. Vet. Microbiol. 157:383-390.

Caporaso, J. G., K. Bittinger, F. D. Bushman, T. Z. DeSantis, G. L. Andersen, and R. Knight. 2010a. PyNAST: A flexible tool for aligning sequences to a template alignment. Bioinformatics $26: 266-267$.

Caporaso, J. G., J. Kuczynski, J. Stombaugh, K. Bittinger, F. D. Bushman, E. K. Costello, N. Fierer, A. G. Pena, J. K. Goodrich, 
and J. I. Gordon. 2010b. QIIME allows analysis of high-throughput community sequencing data. Nat. Methods 7:335-336.

De Vliegher, S., L. K. Fox, S. Piepers, S. McDougall, and H. W. Barkema. 2012. Invited review: Mastitis in dairy heifers: Nature of the disease, potential impact, prevention, and control. J. Dairy Sci. 95:1025-1040.

Derakhshani, H., J. C. Plaizier, and E. Khafipour. 2017. Bacterial ecosystem of the bovine mammary gland: Potential role of foundation taxa in shaping mammary gland microbiota and modulating udder homeostasis. J. Dairy Sci. 100(Suppl. 2):232. (Abstr.)

Derakhshani, H., H. M. Tun, and E. Khafipour. 2016. An extended single-index multiplexed $16 \mathrm{~S}$ rRNA sequencing for microbial community analysis on MiSeq illumina platforms. J. Basic Microbiol. $56: 321-326$.

Dingwell, R. T., K. E. Leslie, Y. H. Schukken, J. M. Sargeant, L. L. Timms, T. F. Duffield, G. P. Keefe, D. F. Kelton, K. D. Lissemore, and J. Conklin. 2004. Association of cow and quarter-level factors at drying-off with new intramammary infections during the dry period. Prev. Vet. Med. 63:75-89.

Doyle, C. J., D. Gleeson, P. W. O'Toole, and P. D. Cotter. 2016. Impacts of seasonal housing and teat preparation on raw milk microbiota: A high-throughput sequencing study. Appl. Environ. Microbiol. 83:e02694-e02616.

Edgar, R. C. 2010. Search and clustering orders of magnitude faster than BLAST. Bioinformatics 26:2460-2461.

Edgar, R. C. 2013. UPARSE: Highly accurate OTU sequences from microbial amplicon reads. Nat. Methods 10:996-998.

Falentin, H., L. Rault, A. Nicolas, D. S. Bouchard, J. Lassalas, P. Lamberton, J.-M. Aubry, P.-G. Marnet, Y. Le Loir, and S. Even. 2016. Bovine teat microbiome analysis revealed reduced alpha diversity and significant changes in taxonomic profiles in quarters with a history of mastitis. Front. Microbiol. 7:480.

Faust, K., and J. Raes. 2012. Microbial interactions: From networks to models. Nat. Rev. Microbiol. 10:538-550.

Fehr, K. B., H. Derakhshani, S. Sepehri, J. C. Plaizier, and E. Khafipour. 2017. Effects of dairy environment on milk microbiota and mammary inflammation. J. Dairy Sci. 100(Suppl. 2):142 (Abstr.).

Ganda, E. K., R. S. Bisinotto, S. F. Lima, K. Kronauer, D. H. Decter, G. Oikonomou, Y. H. Schukken, and R. C. Bicalho. 2016. Longitudinal metagenomic profiling of bovine milk to assess the impact of intramammary treatment using a third-generation cephalosporin. Sci. Rep. 6:37565.

Ganda, E. K., N. Gaeta, A. Sipka, B. Pomeroy, G. Oikonomou, Y. H. Schukken, and R. C. Bicalho. 2017. Normal milk microbiome is reestablished following experimental infection with Escherichia coli independent of intramammary antibiotic treatment with a thirdgeneration cephalosporin in bovines. Microbiome 5:74.

Godden, S., P. Rapnicki, S. Stewart, J. Fetrow, A. Johnson, R. Bey, and R. Farnsworth. 2003. Effectiveness of an internal teat seal in the prevention of new intramammary infections during the dry and early-lactation periods in dairy cows when used with a dry cow intramammary antibiotic. J. Dairy Sci. 86:3899-3911.

Gopal, P. K., and H. Gill. 2000. Oligosaccharides and glycoconjugates in bovine milk and colostrum. Br. J. Nutr. 84:S69-S74.

Halasa, T., O. Østerås, H. Hogeveen, T. Van Werven, and M. Nielen. 2009. Meta-analysis of dry cow management for dairy cattle. Part 1. Protection against new intramammary infections. J. Dairy Sci. 92:3134-3149.

Hammer, O., D. A. T. Harper, and P. D. Ryan. 2012. PAST: Paleontological statistics software package for education and data analysis. Paleontol. Electron. 4 (art. 4):9.

Haveri, M., M. Hovinen, A. Roslöf, and S. Pyörälä. 2008. Molecular types and genetic profiles of Staphylococcus aureus strains isolated from bovine intramammary infections and extramammary sites. J. Clin. Microbiol. 46:3728-3735.

Hornish, R. E., and S. Katarski. 2002. Cephalosporins in veterinary medicine-ceftiofur use in food animals. Curr. Top. Med. Chem. 2:717-731.

Kuehn, J. S., P. J. Gorden, D. Munro, R. Rong, Q. Dong, P. J. Plummer, C. Wang, and G. J. Phillips. 2013. Bacterial community pro- filing of milk samples as a means to understand culture-negative bovine clinical mastitis. PLoS One 8:e61959.

Lima, S. F., A. G. Teixeira, F. S. Lima, E. K. Ganda, C. H. Higgins, G. Oikonomou, and R. C. Bicalho. 2017. The bovine colostrum microbiome and its association with clinical mastitis. J. Dairy Sci. 100:3031-3042.

Looney, W. J., M. Narita, and K. Mühlemann. 2009. Stenotrophomonas maltophilia: An emerging opportunist human pathogen. Lancet Infect. Dis. 9:312-323.

Magoc, T., and S. L. Salzberg. 2011. FLASH: Fast length adjustment of short reads to improve genome assemblies. Bioinformatics 27:2957-2963

Melchior, M. B., H. Vaarkamp, and J. Fink-Gremmels. 2006. Biofilms: A role in recurrent mastitis infections? Vet. J. 171:398-407.

Morgan, X. C., T. L. Tickle, H. Sokol, D. Gevers, K. L. Devaney, D. V. Ward, J. A. Reyes, S. A. Shah, N. LeLeiko, and S. B. Snapper. 2012. Dysfunction of the intestinal microbiome in inflammatory bowel disease and treatment. Genome Biol. 13:R79.

Nam, H. M., S. K. Lim, H. M. Kang, J. M. Kim, J. S. Moon, K. C. Jang, J. M. Kim, Y. S. Joo, and S. C. Jung. 2009. Prevalence and antimicrobial susceptibility of gram-negative bacteria isolated from bovine mastitis between 2003 and 2008 in Korea. J. Dairy Sci. 92:2020-2026.

Nielsen, K. M., P. J. Johnsen, D. Bensasson, and D. Daffonchio. 2007. Release and persistence of extracellular DNA in the environment. Environ. Biosafety Res. 6:37-53.

Ohnishi, M.. T. Sawada, K. Marumo, K. Harada, K. Hirose, A. Shimizu, M. Hayashimoto, R. Sato, N. Uchida, and H. Kato. 2012 Antimicrobial susceptibility and genetic relatedness of bovine Stenotrophomonas maltophilia isolates from a mastitis outbreak. Lett. Appl. Microbiol. 54:572-576.

Oikonomou, G., M. L. Bicalho, E. Meira, R. E. Rossi, C. Foditsch, V. S. Machado, A. G. V. Teixeira, C. Santisteban, Y. H. Schukken, and R. C. Bicalho. 2014. Microbiota of cow's milk; distinguishing healthy, sub-clinically and clinically diseased quarters. PLoS One 9:e85904.

Oliver, S. P., S. E. Murinda, and B. M. Jayarao. 2011. Impact of antibiotic use in adult dairy cows on antimicrobial resistance of veterinary and human pathogens: A comprehensive review. Foodborne Pathog. Dis. 8:337-355.

Patel, S. H., Y. H. Vaidya, C. G. Joshi, and A. P. Kunjadia. 2016. Culture-dependent assessment of bacterial diversity from human milk with lactational mastitis. Comp. Clin. Pathol. 25:437-443.

Paulson, J. N., O. C. Stine, H. C. Bravo, and M. Pop. 2013. Differential abundance analysis for microbial marker-gene surveys. Nat. Methods 10:1200-1202.

Petrovski, K. R., N. B. Williamson, N. Lopez-Villalobos, T. J. Parkinson, and I. G. Tucker. 2011. Culture results from milk samples submitted to veterinary diagnostic laboratories from August 2003 to December 2006 in New Zealand. N. Z. Vet. J. 59:317-322.

Petzer, I. M., D. C. Lourens, T. J. Van der Schans, J. C. Watermeyer, R. Van Reenen, G. H. Rautenbach, and P. Thompson. 2009. Intramammary infection rate during the dry period in cows that received blanket dry cow therapy: Efficacy of 6 different dry-cow intra-mammary antimicrobial products. J. S. Afr. Vet. Assoc. $80: 23-30$.

Pyörälä, S. 2002. New strategies to prevent mastitis. Reprod. Domest. Anim. 37:211-216.

Pyörälä, S. 2008. Mastitis in post-partum dairy cows. Reprod. Domest. Anim. 43:252-259.

Quirk, T., L. K. Fox, D. D. Hancock, J. Capper, J. Wenz, and J. Park. 2012. Intramammary infections and teat canal colonization with coagulase-negative staphylococci after postmilking teat disinfection: Species-specific responses. J. Dairy Sci. 95:1906-1912.

Rabiee, A. R., and I. J. Lean. 2013. The effect of internal teat sealant products (Teatseal and Orbeseal) on intramammary infection, clinical mastitis, and somatic cell counts in lactating dairy cows: A meta-analysis. J. Dairy Sci. 96:6915-6931. 
Stelwagen, K., E. Carpenter, B. Haigh, A. Hodgkinson, and T. T. Wheeler. 2009. Immune components of bovine colostrum and milk. J. Anim. Sci. 87:3-9.

Tang, K. L., N. P. Caffrey, D. B. Nóbrega, S. C. Cork, P. E. Ronksley, H. W. Barkema, A. J. Polachek, H. Ganshorn, N. Sharma, J. D. Kellner, and W. A. Ghali. 2017. Restricting the use of antibiotics in food-producing animals and its associations with antibiotic resistance in food-producing animals and human beings: A systematic review and meta-analysis. Lancet Planet Health 1:e316-e327.

Taponen, S., J. Björkroth, and S. Pyörälä. 2008. Coagulase-negative staphylococci isolated from bovine extramammary sites and intramammary infections in a single dairy herd. J. Dairy Res. 75:422429.

Tena, D., C. Fernández, and M. R. Lago. 2015. Alcaligenes faecalis: An unusual cause of skin and soft tissue infection. Jpn. J. Infect. Dis. $68: 128-130$.

Thomas, C. M., and K. M. Nielsen. 2005. Mechanisms of, and barriers to, horizontal gene transfer between bacteria. Nat. Rev. Microbiol. $3: 711$
Verdier-Metz, I., G. Gagne, S. Bornes, F. Monsallier, P. Veisseire, C. Delbès-Paus, and M.-C. Montel. 2012. Cow teat skin, a potential source of diverse microbial populations for cheese production. Appl. Environ. Microbiol. 78:326-333.

Wei, T. 2017. Corrplot: Visualization of a correlation matrix. R package version 0.84. Comprehensive $\mathrm{R}$ Archive Network. http:// CRAN.R-project.org/package $=$ corrplot

Woodward, W. D., T. E. Besser, A. C. Ward, and L. B. Corbeil. 1987. In vitro growth inhibition of mastitis pathogens by bovine teat skin normal flora. Can. J. Vet. Res. 51:27.

Zhang, R. W. Huo, W. Zhu, and S. Mao. 2015. Characterization of bacterial community of raw milk from dairy cows during subacute ruminal acidosis challenge by high-throughput sequencing. J. Sci. Food Agric. 95:1072-1079. 